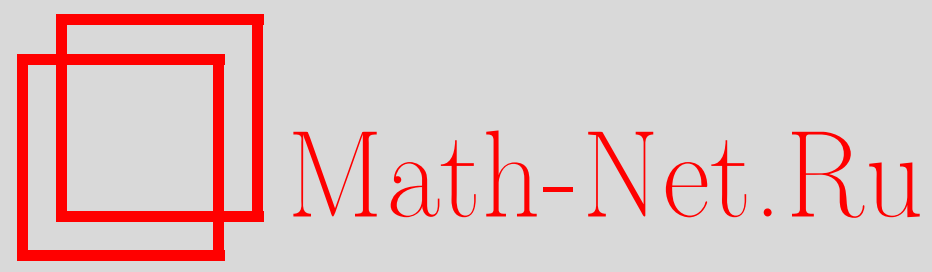

А. А. Борисенко, О локально выпуклых гиперповерхностях в многообразиях Адамара, Матем. заметки, 2000, том 67, выпуск 4, 498-507

DOI: https://doi.org/10.4213/mzm864

Использование Общероссийского математического портала Math-Net.Ru подразумевает, что вы прочитали и согласны с пользовательским соглашением http://www . mathnet.ru/rus/agreement

Параметры загрузки:

IP : 54.197 .130 .99

26 апреля 2023 г., $17: 53: 41$

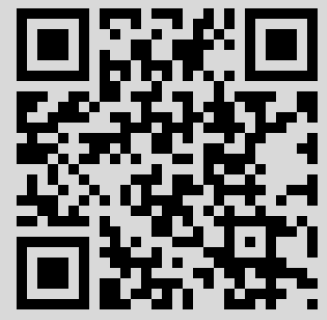




\title{
О ЛОКАЛЬНО ВЫПУКЛЫХ ГИПЕРПОВЕРХНОСТЯХ В МНОГООБРАЗИЯХ АДАМАРА
}

\section{А. А. Борисенко}

\begin{abstract}
Рассматриваются локально выпуклые компактные погруженные гиперповерхности в полом односвязном римановом пространстве неположительной секционной кривизны. Доказывается, что они есть выпуклые гиперповерхности, гомеоморфиные сфере. Доказывается аналогичный результат для погруженных гиперповерхностей с неположительно определенной второй квадратичной формой, ранг которой не меньше единицы.

Библиография: 12 названий.
\end{abstract}

Ж. Адамар доказал следующую теорему. Пусть $\varphi$ - погружение компактного ориентированного $n$-мерного многообразия в евклидово пространство $E^{n+1}(n \geqslant 2)$ со всюду положительной гауссовой кривизной. Тогда $F(M)$ есть выпуклая гиперповерхность [1].

Чжень и Лашоф [2] обобшили эту теорему. Пусть $\varphi$ - погружение компактного ориентированного $n$-мерного многообразия $M$ в $E^{n+1}$. Тогда следующие два утверждения эквивалентны:

1) степень сферического отображения равна \pm 1 , и гауссова кривизна не меняет знака (т.е. всюду $\geqslant 0$ или $\leqslant 0)$;

2) $\varphi(M)$ есть выпуклая гиперповерхность.

Под гауссовой кривизной мы понимаем здесь произведение главных кривизн.

Топологическое погружение $f: N^{n} \rightarrow M$ многообразия $N^{n}$ в риманово многообразие $M$ называется локально выпуклым в точке $x \in N^{n}$, если у $x$ есть окрестность $U$ такая, что $f(U)$ является частью границы вьпуклого множества в $M$.

Хейенорт доказал следующую теорему. Пусть $f: N^{n} \rightarrow E^{n+1}, n \geqslant 2$, - топологическое погружение связного многообразия $N^{n}$. Если $f$ локально вьпукло во всех точках и имеет хотя бы одну точку локальной строгой опорности, а $N^{n}$ полно в индуцированной этим погружением метрике, то $f$ есть вложение, а $F=f\left(N^{n}\right)$ - граница вьпуклого тела [3].

В пространстве Лобачевского для $h$-локально выпуклых (т.е. таких, что в каждой точке гиперповерхности есть окрестность, которая лежит по одну сторону орисферы) регулярньх гиперповерхностей эта теорема обобщалась в [4], для нерегулярных гиперповерхностей - в [5].

Наша цель обобщить эту теорему для компактного случая, когда объемлющим пространством является полное односвязное риманово многообразиенеположительной секционной кривизны. Такое многообразие мы будем называть многообразием Адамара. 
Пусть $M$ есть полное риманово многообразие. Ниже используем следующие определения [6].

1. Множество $A \subset M$ назьвается абсолютно выпуклым, если каждая геодезическая с концами в $A$ целиком содержится в $A$.

2. $A \subset M$ назьвается выпуклым, если каждая кратчайшая с концами в $A$ содержится B $A$.

3. $A \subset M$ называется локально выпуклым, если каждая точка $P \in A$ имеет в $M$ окрестность $U_{P}$, для которой множество $U_{P} \cap A$ вьпукло.

4. Множество $A \subset M, A \neq M$, назьвается строго гранично выпуклым, если у $A$ в каждой граничной точке есть “внешняя вогнутая опора" и, кроме того, $A$ - локально выпукло.

Топологическое погружение $f: N^{n} \rightarrow M$ многообразия $N^{n}$ в риманово многообразие $M$ называется локально выпуклым в точке $x \in N^{n}$, если у $x$ есть окрестность $U$ такая, что $f(U)$ является частью границы вьпуклого множества в $M$.

Теорема 1. Пусть $f: N^{n} \rightarrow M^{n+1}, n \geqslant 2$, есть погружение компактного связного многообразия $N^{n}$ в полное односвязное риманово многообразие неполоэительной секционной кривизны $M^{n+1}$. Если погружение $f$ локально выпукло, то $f$ есть влохсение, а $f\left(N^{n}\right)=F$ есть граница выпуклого мнохества в $M^{n+1} u$ $f\left(N^{n}\right)=F$ гомеоморфно сфере $S^{n}$.

Лемма 1 [6]. В многообразии Адамара у непустого выпуклого множсества $B$, отличного от $M$, внешние әквидистантные множества $B_{\rho}:=\{x \in M \mid d(x, B) \leqslant \rho\}$ выпуклы, здесь $d(x, B)$ - расстояние точки от множества $B$.

Граница эквидистантного множества называется внешней эквидистантной поверхностью или просто внешней эквидистантой.

ЛЕмма 2 [7]. Достаточно близкие к В внешние эквидистанты являются $C^{1,1}$ гладкими поверхностями.

Лемма 3 [6]. В многообразии Адамара иары любого радиуса абсолютно выпукль, а ограничивающие их сферы являются выпуклыми гиперповерхностями, диффеоморфными стандартной сфере. Главные кривизны сферы радиуса г удовлетворяют неравенству $K \geqslant 1 / r$.

ЛЕмма 4 [8]. Для любых геодезических $\gamma_{1}(t), \gamma_{2}(t):[0,1] \rightarrow M$ в пространстве Адамара функиия $f(t)=\left|\gamma_{1}(t), \gamma_{2}(t)\right|$ выпукла.

Лемма 5 [6]. Пусть $\Omega$ открытое, но не обязательно связное мнохсество в $M$, ограниченное в $M$ гладкой связной гиперповерхностью $F$, все главные кривизны которой по отношению $к$ направленным в $\Omega$ нормалям положстельны. Тогда множества $\Omega$ и $\bar{\Omega}$ являются строго гранично выпуклыми.

Лемма 6 [9]. Пусть $F$ - гиперповерхность риманова многообразия $M, \widetilde{F}-$ - иперповерхность риманова многообразия $\widetilde{M}$. Пусть $\gamma:[0, a] \rightarrow M, \widetilde{\gamma}:[0, a] \rightarrow \widetilde{M}-$ геодезические, перпендикулярные $F$ и $\widetilde{F}$ соответственно, с начальными точками $P \in F, \widetilde{P} \in \widetilde{F}$ и параметризованные длиной дуги. 
Пусть выполняются следующие условия:

1) для каждого $t \in[0, a]$ и любых двумерных направлений $\sigma \in T_{\gamma(t)} M, \widetilde{\sigma} \in T_{\bar{\gamma}(t)} \widetilde{M}$, содержащих векторы $\gamma^{\prime}(t) \in \sigma, \bar{\gamma}^{\prime}(t) \in \widetilde{\sigma}$, выполняется неравенство $K_{\sigma} \leqslant K_{\tilde{\sigma}}$, әде $K_{\sigma}, K_{\widetilde{\sigma}}$ - секционные кривизны в направлении площадок $\sigma, \widetilde{\sigma}$;

2) наибольиая главная кривизна гиперповерхности $F$ не превосходит наименьией главной кривизны $\widetilde{F}$ соответственно в точках $P=\gamma(0), P=\widetilde{\gamma}(0)$, т.е. $k_{\max } \leqslant \widetilde{k}_{\min }$

3) на $\widetilde{\gamma}$ нет фокальных точек для $\widetilde{P}=\widetilde{\gamma}(0)$.

Тогда на ү нет фокальных точек для $P=\gamma(0)$.

ЛЕмма 7. Пусть $F$ есть гиперповерхность с неполохительно определенной второй квадратичной формой в многообразии Адамара относительно единичного нормального векторного поля $\xi$. Тогда параллельная гиперповерхность $F_{\rho}$ со сдвигом в направлении нормального векторного поля $\xi$ также имеет неположительную вторую квадратичную форму.

ДокАЗАТЕЛЬСТво лЕммы 7. Соответствующие нормальные кривизны параллельных гиперповерхностей удовлетворяют уравнению [10]

$$
k^{1}=k^{2}+f(t)
$$

где $f(t) \leqslant 0$ в силу неположительности секционной кривизны объемлющего пространства, $k(0) \leqslant 0$ в силу неположительной определенности второй квадратичной формы.

Если положить $k=-u^{\prime} / u$, то $u$ удовлетворяет уравнению

$$
u^{\prime \prime}+f(t) u=0
$$

при начальных условиях $u(0)=1, u^{\prime}(0)=-k(0) \geqslant 0$. Отсюда следует, что $u$-выпуклая функция, $u^{\prime}(t) \geqslant 0, u>0$ и нормальные кривизны $k(t) \leqslant 0$.

ДОКАЗАТЕЛЬСТВо ТЕОРЕМЫ 1 . Пусть $F=f(N)$ - локально выпуклое погружение $n$-мерного компактного многообразия $N$ в $(n+1)$-мерное полное односвязное риманово многообразие $M$ неположительной секционной кривизны. Несмотря на то, что мы имеем только погружение, а не вложение, мы можем определить внешнюю эквидистанту $f(N)=F$. Обозначим ее $F_{\rho}$. По лемме $1 F_{\rho}$ является локально выпуклой гиперповерхностью, и по лемме 2 она имеет гладкость класса $C^{1,1}$. Пусть $S$ - сфера в $M$ достаточно большого радиуса такого, что шар, которьй ограничивает эта сфера, содержит $f(N)$ и $F_{\rho}$ при достаточно малом $\rho$.

В каждой точке $F_{\rho}$ есть внешняя нормаль $\xi$. Выпустим в этом направлении геодезический луч до пересечения со сферой $S$. Тем самым, мы зададим отображение

$$
\nu: F_{\rho} \rightarrow S
$$

Покажем, что это накрытие.

Пусть $P_{1}, Q_{1}$ две точки $F_{\rho}$, которые лежат на одной локально вьпуклой гиперповерхности, $\gamma_{1}, \gamma_{2}$ - геодезические лучи, вьпущенные в направлении внешних нормалей из точек $P_{1}, Q_{1} ; P_{2}, Q_{2}$ - точки пересечения лучей с гиперсферой $S$. Заметим, что величина углов $\angle Q_{1} P_{1} P_{2}$ и $\angle P_{1} Q_{1} Q_{2}$ не меньше $\pi / 2$, так как в силу локальной вьпуклости 
$F_{\rho}$ кратчайшая $P_{1} P_{2}$ проходит внутри внешнего эквидистантного множества (не исключая выхода на границу).

Применим лемму 4 к случаю, когда $\gamma_{1}=P_{1} P_{2}, \gamma_{2}=Q_{1} Q_{2}, f(t)=|P(t) Q(t)|$, где $P(t) \in \gamma_{1}, Q(t) \in \gamma_{2}$. В силу формулы первой вариации длины и оценок на углы $f^{\prime}(0) \geqslant 0$. Поэтому из вьпуклости $f$ следует, что $\left|P_{2} Q_{2}\right| \geqslant\left|P_{1} Q_{1}\right|$. Отсюда следует, что локально различные точки переходят в различные точки. Так как $F_{\rho}$ имеет регулярность класса $C^{1,1}$, то если $Q_{1} \stackrel{\rho}{\rightarrow} P_{1}$, то $Q_{2} \rightarrow P_{2}$. Поэтому отображение $\nu: F_{\rho} \rightarrow S$ локально является топологическим отображением и, следовательно, $\nu$ есть накрытие. Так как при $n>1$ сфера является односвязной, то эквидистанта $F_{\rho}$ гомеоморфна $S$ и этот гомеоморфизм задается отображением $\nu$.

Вне шара $D$ нормальные лучи к $F_{\rho}$ не могут пересекаться. Допустим противное. Пусть лучи из точек $P_{1}, Q_{1}$ пересекаются в точке $Q$, которая лежит вне шара $D$. Проведем через $Q$ сферу $S_{Q}$, концентричную $S$, с тем же центром $O$. И зададим то же отображение $\nu$. Тогда отображение $\nu$ на сферу $S_{Q}$ не является гомеоморфизмом. Это противоречит вьше доказанному.

Возьмем поверхность $F_{\rho+t_{0}}$, параллельную $F_{\rho}$, так, чтобы она лежала вне шара $D$. Локально она является эквидистантой $F$, поэтому она локально вьпукла. Кроме того, $F_{\rho+t_{0}}$ уже является вложенной поверхностью, поверхностью без самопересечений, а также границей некоторого тела.

Аналогично доказательству теоремы Титце мы получим, что тело, ограниченное поверхностью $F_{\rho+t_{0}}$, выпукло, а $F_{\rho+t_{0}}$ является компактной вьпуклой гиперповерхностью, гомеоморфной сфере. Теперь берем поверхности $F_{\rho+t_{0}-t}$, параллельные $F_{\rho+t_{0}}$ и лежащие внутри тела $D_{\rho+t_{0}}$, ограниченного $F_{\rho+t_{0}}$. При малых $t$ поверхность $F_{\rho+t_{0}-t}$ является вложенной, локально выпуклой при всех $t<\rho+t_{0}$, а значит, глобально выпуклой. Обозначим через $t_{1}$ точную верхнюю грань таких $t\left(0 \leqslant t \leqslant \rho+t_{0}\right)$, что поверхность $F_{\rho+t_{0}-t}$ является вложенной, а значит, и выпуклой поверхностью. Пусть $t_{1}<\rho+t_{0}$. Тело $D_{\rho+t_{0}-t_{1}}$, ограниченное $F_{\rho+t_{0}-t_{1}}$, является вьпукльг телом, a $F_{\rho+t_{0}}-t_{1}$ является выпуклой поверхностью. На ней должны быть точки самокасания $F_{\rho+t_{0}-t_{1}}$. Возможны два случая самокасания: внутреннее и внешнее. Первый случай невозможен, так как при внутреннем самокасании отображение $\nu$ не было бы гомеоморфизмом. Второй случай невозможен из-за вьпуклости тела $D_{\rho+t_{0}}-t_{1}$. Отсюда следует, что $t_{1}=\rho+t_{0}$ и $F$ является границей абсолютно выпуклого тела. Взяв точку внутри тела, мы покажем, что гиперповерхность $F$ гомеоморфна сфере.

Пусть $f: N^{n} \rightarrow M^{n+1}$ есть погружение класса $C^{2}$ в риманово пространство $M^{n+1}$. Тогда в каждой точке можно определить гауссову кривизну как произведение главных кривизн: $K=k_{1} k_{2} \cdots k_{n}$.

Как следствие теоремы 1 мы получим обобщение теоремы Адамара.

Теорема 2. Пусть $f: N^{n} \rightarrow M^{n+1}, n \geqslant 2$, - регулярное класса $C^{2}$ погружсние компактного связного многообразия $N^{n}$ в полное односвязное риманово многообразие неположительной секиионной кривизны. Если гауссова кривизна положительна, то $F=f\left(N^{n}\right)$ есть выпуклая гиперповерхность, гомеоморфная сфере.

ДокАЗАТЕЛЬСтво. Пусть $S$ - сфера, описанная вокруг гиперповерхности $f\left(N^{n}\right)=F$, ее радиус равен $r, P$ - точка соприкосновения. Тогда в окрестности $P$ поверхность $F$ лежит внутри сферы $S$. Ее главные кривизны в точке $P$ не менњше, чем главные кривизны 
сферы в этой точке. По лемме 3 для сферы они оцениваются снизу через $1 / r$, где $r$ - радиус сферы. Поэтому для $F$ в точке $P$ главные кривизны $k_{i} \geqslant 1 / r$ и они одного знака. В силу знакопостоянства гауссовой кривизны $K$ главные кривизны нигде не обращаются в нуль. Поэтому во всех точках $F$ главные кривизны одного знака. Тогда из леммы 5 следует, что $F$ является локально выпуклой гиперповерхностью. Применяя теорему 1 , мы получим утверждение теоремы 2.

Теорема 3. Пусть $f: N^{n} \rightarrow M^{n+1}, n \geqslant 2$, - регулярное класса $C^{2}$ погружсене компактного связного многообразия $N^{n}$ в полное односвязное риманово многообразие неположстельной секчионной кривизны. Если в каждой точке вторая квадратичная форма при выборе соответствующей нормали неполохительно определена и ранг второй квадратичной формы $\geqslant 1$, то $F=f\left(N^{n}\right)$ есть выпуклая гиперповерхность, диффеоморфная сфере.

ЗАмЕчАниЕ. Когда работа была сдана в печать, автор узнал, что теорема 3 фактически доказана С. Александер в [11], но предлагаемое доказательство основано на другой идее, которая дает подход к решению проблемы без условия на ранг второй квадратичной формы.

Нам кажется, что теорема 3 справедлива без условия на ранг второй квадратичной формы.

ДоКАЗАТЕЛЬСТВО ТЕОРЕМЫ 3. Выберем в каждой точке гиперповерхности $F=$ $f\left(N^{n}\right)$ единичную нормаль таким образом, чтобы вторая квадратичная форма погруженной гиперповерхности $F$ относительно этой нормали была неположительно определена. В силу условия на ранг второй квадратичной формы такая нормаль определяется единственным образом. Таким образом, на $F$ мы получили единичное поле нормалей $\xi$. Возьмем сферу $S$ достаточно большого радиуса $r$ такую, что погруженная гиперповерхность $F$ лежит внутри шара $D$, ограниченного сферой $S$. Вьпустим из точек $F$ в направлении единичных нормалей $\xi$ геодезические лучи $\gamma(\xi)$. Из леммы 6 следует, что на этих геодезических лучах нет фокальных точек. В качестве пространства сравнения $\widetilde{M}$ берем евклидово пространство. Поэтому отображение $\nu:(0, \infty) \times F \rightarrow M$, когда нормали $t \xi, t>0$, ставится в соответствие точка $\exp t \xi$ на геодезическом луче $\gamma(\xi)$, является локальньг гомеоморфизмом.

Оценим угол, которьй образуют лучи $\gamma(\xi)$ с внешними нормалями сферы $S$ достаточно большого радиуса в точке пересечения. Погруженная гиперповерхность $F=f\left(N^{n}\right)$ содержится внутри шара, ограниченного этой сферой. Пусть расстояние точек $P \in F$ от точек сферы $\geqslant r_{0}$, радиус сферы равен $r$. Рассмотрим $\triangle P O Q$, где $O$ - центр сферы, $P \in F, Q$ - точка пересечения луча $\gamma(\xi)$ со сферой. Этот луч выходит из точки $P$. Тогда $|P Q| \geqslant r_{0},|O P| \leqslant r,|O Q|=r$, где $r-$ радиус сферы, $\alpha=\angle P Q O$.

Рассмотрим на евклидовой плоскости треугольник $\triangle \bar{P} \bar{O} \bar{Q}$ с теми же длинами сторон. По теореме сравнения $\angle \bar{P} \bar{Q} \bar{O}=\bar{\alpha} \geqslant \alpha=\angle P Q O$. Оценим угол $\bar{\alpha}$. Поскольку $|\bar{P} \bar{O}|^{2}=r^{2}+|\bar{P} \bar{Q}|^{2}-2 r|\bar{P} \bar{O}| \cos \bar{\alpha}$, то

$$
\cos \bar{\alpha}=\frac{r^{2}+|\bar{P} \bar{Q}|^{2}-|\bar{P} \bar{O}|^{2}}{2 r|\bar{P} \bar{Q}|} \geqslant \frac{r_{0}}{2 r} .
$$

Так как $\cos \alpha \geqslant \cos \bar{\alpha}$, то $\cos \alpha \geqslant r_{0} /(2 r), \alpha \leqslant \arccos r_{0} /(2 r)$. Из этого неравенства и из факта отсутствия фокальных точек следует, что локально отображение $\nu: F \rightarrow S$, 
когда точке $P \in F$ ставится в соответствие точка $Q$ пересечения геодезического луча $\gamma(\xi)$ со сферой $S$, является гомеоморфизмом.

Отсюда вытекает, что отображение $\nu$ является накрытием. Из односвязности сферы следует, что отображение $\nu$ задает гомеоморфизм $F$ на $S$. Отсюда параллельные поверхности $F_{\rho}$ при достаточно большом $\rho$ являются вложенными гиперповерхностями.

Допустим, что вложенная гиперповерхность $F_{\rho}$ невьпуклая. Возьмем выпуклую оболочку такой гиперповерхности. Пусть $\bar{G}$ - связная область на выпуклой оболочке $\bar{F}_{\rho}$, которая не лежит на гиперповерхности $F_{\rho}, G$ - соответствующая по гомеоморфизму на $S$ невьпуклая область, имеющая с $\bar{G}$ общую границу. Через каждую внутреннюю точку области $\bar{G}$ проходит отрезок кратчайшей объемлющего пространства, который полностью принадлежит $\bar{G}$, с концами на границе области. Область $G$ лежит внутри вьпуклой оболочки. Отображение по лучам $\gamma(\xi)$, когда ставятся в соответствие точки пересечения луча с $G$ и $\bar{G}$, задает гомеоморфизм этих областей. Возьмем внешние эквидистанты $G_{\rho+t}$ области $G$. Найдется такое значение $t_{0}$, что $G_{\rho+t_{0}}$ лежит вне внутренности тела, ограниченного выпуклой оболочкой $\bar{F} \rho$, но имеет общие точки с внутренностью области $\bar{G}$. Пусть это будет точка $P_{0} \in \bar{G} \cap G_{\rho+t_{0}}$.

В точке $P_{0}$ относительно внешней нормали $\xi$ вторая квадратичная форма неположительно определенная. Это следует из леммы 7. Отрезок кратчайшей $l$, которьй лежит на $\bar{G}$, имеет концы на границе области $G_{\rho}$. Поэтому своими концами он уходит внутрь тела, ограниченного гиперповерхностью $F_{\rho+t_{0}}$.

Внешняя нормаль $\xi$ к поверхности $G_{\rho+t_{0}}$ в точке $P_{0}$ одновременно будет нормалью к выпуклой оболочке $F_{\rho}$, касательньй вектор геодезической $l$ будет лежать в касательной плоскости. Параллельно перенесем $\xi$ в объемлющем пространстве и в направлении векторного поля $\xi(t)$, где $t$ - параметр длины на $l$, выпустим геодезические. Мы получим двумерную поверхность $F^{2}$, которая пересекает поверхность $G_{\rho+t_{0}}$ по регулярной кривой $L$. Это есть аналог нормального сечения. На поверхности $F^{2}$ кривая $L$ лежит по одну сторону геодезической $l$ в полуплоскости, куда направлена нормаль $\xi$, и полностью не совпадает $\mathrm{c} l$. Не ограничивая общности, будем считать, что при $t>0$ точки $L$ лежат выше геодезической $l$. Возьмем на $L$ точку $P$, лежащую справа от точки $P_{0}$. Проведем отрезок кратчайшей $l_{1}$, соединяющий эти точки. Вместе с дугой $P_{1} P_{2}$ кривой $L$ этот отрезок ограничивает на $F^{2}$ односвязную область $\alpha$ (может быть, это будет меньший отрезок). Это аналог отрезания шапочки в евклидовом пространстве.

По теореме Гаусса-Бонне для области $\alpha$ выполняется соотношение

$$
\int_{l_{1}} k_{g} d s=-\int K d S+\alpha_{1}+\alpha_{2},
$$

где $K$ - гауссова кривизна $F^{2}, k_{g}$ - геодезическая кривизна кривой $L, \alpha_{1}>0, \alpha_{2}>0$ - углы между кривой $L$ и геодезической $l_{1}$. Поверхность $F^{2}$ - линейчатая. Поэтому ее гауссова кривизна не больше кривизны объемлющего пространства. Отсюда $K \leqslant 0$ и $\int_{l_{1}} k_{g} d s>0$. Значит, найдется точка на кривой $L$, геодезическая кривизна которой на $F^{2}$ больше нуля. И такая точка найдется в сколь угодно малой окрестности точки $P$.

Введем в объемлющем пространстве вдоль геодезической $l$ систему координат Ферми следуюшим образом: на геодезической $l$ введем параметризацию $l(t)$ с помощью длины дуги, отсчитьваемой от $P_{0}$ вдоль $l$. Пусть $\left\{e_{i}\right\}_{i=1}^{n+1}$ есть ортонормированньй репер в точке $P_{0}$, причем вектор $e_{1}$ касается геодезической $l, e_{2}$ направлен по нормали $\xi$. Обозначим $e_{i}(t)$ векторы, полученные из векторов $e_{i}$ путем параллельного переноса вдоль $l$ 
в точку $l(t)$. Пусть $Q$-произвольная точка объемлющего пространства $M^{n+1}$ вблизи $l$. Найдем на $l$ точку $l\left(t_{0}\right)$, ближайшую $Q$. Соединим $Q$ и $l\left(t_{0}\right)$ кратчайшей. Обозначим через $\eta^{i}$ координаты единичного вектора, касательного к кратчайшей $l\left(t_{0}\right) Q$ в точке $l\left(t_{0}\right)$ относительно репера $\left\{e_{i}\left(t_{0}\right)\right\}$. Положим $x^{1}=t_{0}, x^{i}=\eta^{i}\left|l\left(t_{0}\right) Q\right|$, где $\left|l\left(t_{0}\right) Q\right|$ - длина отрезка геодезической.

Полученная таким образом система координат есть нормированная система координат Ферми. В этой системе координат вдоль $l$ коэффициенты метрического тензора $g_{i j}=\delta_{i j}$ и символы Кристоффеля $\Gamma_{i j}^{k}=0$.

Поверхность $F^{2}$ в этой системе координат задается уравнением $x^{3}=\cdots=x^{n+1}=0$. В доль геодезической $l$ коэффициенты вторых квадратичных форм $F$ имеют вид:

$$
b_{i j}^{\alpha}=\frac{1}{2} \frac{\partial g_{i j}}{\partial x^{3}} ; \quad \alpha=3, \ldots, n+1 ; \quad i, j=1,2 .
$$

Они равны нулю вдоль $l$.

Пусть для кривой $L$ геодезическая кривизна $k_{g}>0$ в точке $P_{0}$. В силу вполне геодезичности $F^{2}$ вдоль $l$ вектор главной кривизны $L$ как кривой в объемлющем пространстве $M^{n+1}$ совпадает с вектором главной кривизны $L$ как кривой на $F^{2}$, и он совпадает с нормалью $\xi$. Геодезическая кривизна $k_{g}$ совпадает с нормальной кривизной гиперповерхности $G_{\rho+t_{0}}$ в точке $P_{0}$ в направлении $l$. Поэтому нормальная кривизна положительна в этом направлении. Но это противоречит неположительной определенности второй квадратичной формы $G_{\rho+t_{0}}$ относительно нормали $\xi$. Рассмотрим второй случай, когда $k_{g}=0$ в точке $P_{0}$. Тогда в окрестности $P_{0}$ кривая $L$ задается в виде:

$$
\begin{gathered}
x^{2}=f\left(x^{1}\right) ; \quad f>0, \quad \text { если } x^{1}>0 ; \\
f(0)=f^{\prime}(0)=f^{\prime \prime}(0)=0 ; \quad x^{3}=\cdots=x^{n+1}=0 .
\end{gathered}
$$

Тогда существует последовательность точек $P_{n} \rightarrow P$ на $L$ с координатой $x_{n}^{1} \rightarrow 0$ таких, что

$$
\lim _{x_{n}^{1} \rightarrow 0} \frac{f^{\prime \prime}\left(x_{n}^{1}\right)}{f}=+\infty .
$$

Пусть в точках $P_{n} \rightarrow P$ геодезическая кривизна $k_{g}>0$. Вектор главной кривизны $\nu_{1}$ кривой $L$ как кривой на $F^{2}$ имеет координаты

$$
\left\{\begin{array}{l}
Y^{\sigma}=\frac{d^{2} x^{\sigma}}{d s^{2}}+\Gamma_{i j \backslash a}^{\sigma} \frac{d x^{i}}{d s} \frac{d x^{j}}{d s} \\
Y^{\alpha}=0
\end{array}\right.
$$

где $i, j, \sigma=1,2 ; \alpha=3, \ldots, n+1 ; \Gamma_{i j \backslash a}^{\sigma}-$ символы Кристоффеля метрики $F^{2} ; s-$ длина кривой $L$.

Вектор главной кривизны $\nu$ кривой $L$ как кривой в римановом многообразии $M^{n+1}$ имеет вид:

$$
\left\{\begin{array}{l}
Y^{\sigma}=\Gamma_{i j \backslash g}^{\sigma} \frac{d x^{i}}{d s} \frac{d x^{j}}{d s} \quad(\sigma=1,2) ; \\
Y^{\alpha}=\Gamma_{i j \backslash g}^{\alpha} \frac{d x^{i}}{d s} \frac{d x^{j}}{d s} \quad(\alpha=3, \ldots, n+1),
\end{array}\right.
$$


где $\Gamma_{i j \backslash g}^{\sigma}, \Gamma_{i j \backslash g}^{\alpha}-$ символы Кристоффеля метрики объемлющего пространства. Координаты единичного касательного вектора к $L$ имеют вид

$$
\left(\frac{d x^{1}}{d s}, \frac{d x^{2}}{d s}, 0, \ldots, 0\right), \quad\left(\frac{d x^{1}}{d s}(0)\right)=1, \quad\left(\frac{d x^{2}}{d s}(0)\right)=0,
$$

и для них вьполняется соотношение

$$
g\left(\frac{d x^{1}}{d s}\right)^{2}+\left(\frac{d x^{2}}{d s}\right)^{2}=1
$$

где $d s^{2}=g\left(d x^{1}\right)^{2}+\left(d x^{2}\right)^{2}$ есть первая квадратичная форма поверхности $F^{2}$.

В точках $P_{n}$ кривой $L$ геодезическая кривизна $k_{g}$ на поверхности $F^{2}$ имеет порядок $k_{g} \approx f^{\prime \prime}\left(x^{1}\right)$. Это непосредственно следует из формулы для геодезической кривизны.

Символы Кристоффеля имеют порядок

$$
\Gamma_{i j \backslash a}^{\sigma}\left(x^{1}, x^{2}\right) \approx 0\left(f\left(x_{1}\right)\right) .
$$

Подставим в (5) $x^{2}=f\left(x^{1}\right)$. Мы получим

$$
\begin{gathered}
g\left(\frac{d x^{1}}{d s}\right)^{2}+\left(f\left(x^{1}\right)\right)^{2}\left(\frac{d x^{1}}{d s}\right)^{2}=1 \\
\left(\frac{d x^{1}}{d s}\right)^{2}=\frac{1}{g+\left(f^{\prime}\left(x_{1}\right)\right)^{2}} ; \quad \frac{d x^{2}}{d s}=f^{\prime}\left(x_{1}\right) \frac{d x^{1}}{d s} .
\end{gathered}
$$

Продифференцируем (5) по $s$. Имеем

$$
\begin{gathered}
\left(\frac{\partial g}{\partial x^{1}} \frac{\partial x^{1}}{\partial s}+\frac{\partial g}{\partial x^{2}} f^{\prime}\left(x^{1}\right) \frac{d x^{1}}{d s}\right)\left(\frac{d x^{1}}{d s}\right)^{2}+2 g \frac{d x^{1}}{d s} \frac{d^{2} x^{1}}{d s^{2}} \\
+2 \frac{d x^{2}}{d s} f^{\prime \prime}\left(x^{1}\right)\left(\frac{d x^{1}}{d s}\right)^{2}+2 \frac{d x^{2}}{d s} f^{\prime}\left(x^{1}\right) \frac{d^{2} x^{1}}{d s^{2}}=0
\end{gathered}
$$

Отсюда

$$
\frac{d^{2} x^{1}}{d s^{2}}\left(2 g \frac{d x^{1}}{d s}+2 \frac{d x^{1}}{d s}\left(f^{\prime}\right)^{2}\right)+\left(\frac{d g}{d x^{1}} \frac{d x^{1}}{d s}+\frac{d g}{d x^{2}} f^{\prime} \frac{d x^{1}}{d s}\right)\left(\frac{d x^{1}}{d s}\right)^{2}+2 f^{\prime} f^{\prime \prime}\left(\frac{d x^{1}}{d s}\right)^{3}=0
$$

Учитьвая, что в окрестности точки $P_{0} \partial g / \partial x^{1} \approx f\left(x^{1}\right) ; \partial g / \partial x^{2} \approx f\left(x^{1}\right)$, мы получим, что

$$
\begin{aligned}
& \frac{d^{2} x^{1}}{d s^{2}} \approx f+\frac{1}{2} f f^{\prime}+f^{\prime} f^{\prime \prime} \\
& \frac{d^{2} x^{2}}{d s^{2}}=f^{\prime \prime}\left(\frac{d x^{1}}{d s}\right)+f^{\prime} \frac{d^{2} x^{1}}{d s^{2}} \approx f^{\prime \prime} .
\end{aligned}
$$

Из (3) следует, что координаты вектора $\nu_{1}$ имеют порядок

$$
\left\{\begin{array}{l}
Y^{1} \approx f+f^{\prime} f^{\prime \prime} ; \\
Y^{2} \approx f^{\prime \prime} ; \\
Y^{\alpha} \approx 0 \quad(\alpha=3, \ldots, n+1) .
\end{array}\right.
$$


Из (2) следует, что в точках $P_{n} \lim _{P_{n} \rightarrow P_{0}} Y^{1} / Y^{2}=0$, т.е. вектор главной нормали $\nu_{1}\left(P_{n}\right)$ стремится к направлению вектора нормали $\xi$ в точке $P_{0}$. Отсюда вытекает, что при достаточно большом $n$ вектор $\nu_{1}\left(P_{n}\right)$ образует мальй угол с внешней нормалью $\xi\left(P_{n}\right)$ гиперповерхности $G_{\rho+t_{0}}$, которьй стремится к нулю при $P_{n} \rightarrow P_{0}$.

Аналогично из (4) следует, что координаты вектора главной кривизны $\nu$ кривой $L$ как кривой в объемлющем римановом пространстве имеют порядок

$$
\left\{\begin{array}{l}
Y^{1} \approx f+f^{\prime} f^{\prime \prime} \\
Y^{2} \approx f^{\prime \prime} \\
Y^{\alpha} \approx f
\end{array}\right.
$$

Из (2) также вытекает, что вектор $\nu$ при $P_{n} \rightarrow P_{0}$ стремится к направлению вектора нормали $\xi$ в точке $P_{0}$. Отсюда следует, что $\nu\left(P_{n}\right)$ образует малый угол, стремящийся $\mathrm{K}$ нулю при $P_{n} \rightarrow P_{0}$, с внешней нормалью $\xi\left(P_{n}\right)$ гиперповерхности $G_{\rho+t_{0}}$, относительно которой вторая квадратичная форма гиперповерхности неположительно определенная.

В точках $P_{n}$ вектор $\nu_{1}\left(P_{n}\right)$ отличен от нуля. Вектор $\nu\left(P_{n}\right)$ есть ортогональная проекция вектора $\nu\left(P_{n}\right)$ на касательную плоскость к поверхности $F^{2}$, поэтому он также отличен от нуля. Так как угол между $\nu\left(P_{n}\right)$ и единичной нормалью $\xi\left(P_{n}\right)$ мальй, то нормальная кривизна гиперповерхности $G_{\rho+t_{0}}$ в направлении вектора касательной кривой $L$ в точке $P_{n}$ больше нуля при достаточно большом $n$. Это противоречит неположительной определенности второй квадратичной формы.

Тем самым, предположив, что $F_{\rho}$ невьпуклая гиперповерхность, мы пришли к противоречию. Дальше, следуя ходу доказательства теоремы 1 , отступая назад, мы докажем выпуклость гиперповерхности $F$.

Пусть $\sigma$ есть двумерная площадка, касательная к гиперповерхности $F$. Внешней кривизной в направлении площадки $\sigma$ называется

$$
K(\sigma)=K(\sigma)_{\backslash F}-K(\sigma)_{\backslash M},
$$

где $K(\sigma)_{\backslash F}$ - секционная кривизна гиперповерхности $F$ в направлении этой площадки, $K(\sigma) \backslash M-$ секционная кривизна объемлющего пространства $M$.

Если для компактной погруженной гиперповерхности во всех точках и всех двумерных направлениях внешняя секционная кривизна положительна, то из формулы Гаусса следует, что вторая квадратичная форма будет знакоопределена, и из теоремы 3 следует, что гиперповерхность есть выпуклая гиперповерхность, диффеоморфная сфере.

Пусть $\tau$ есть касательньй вектор к гиперповерхности $F, R(\tau) / F-$ кривизна Риччи гиперповерхности $F$ в направлении $\tau, R(\tau) / M$ - ограничение кривизны Риччи объемлющего пространства на касательное пространство к $F$, т.е.

$$
\operatorname{Ric} \tau_{/ M}=\sum_{i=2}^{n} K\left(\tau, e_{i}\right)_{/ M}
$$

где $\tau, e_{i}(i=2, \ldots, n)$ - ортонормированный репер касательного пространства к $F$, $K\left(\tau, e_{i}\right)$ - секционные кривизны $M$ по площадкам, натянутым на векторы $\tau, e_{i}$.

Внешняя кривизна Риччи поверхности $F$ в направлении $\tau$ определяется равенством

$$
\overline{\operatorname{Ric}} \tau=\operatorname{Ric} \tau / F-\operatorname{Ric} \tau / M
$$


Внешняя скалярная кривизна поверхности $F$ определяется равенством:

$$
\bar{R}=R_{/ F}-R_{/ M}
$$

где $R_{/ F}-$ скалярная кривизна $F$, а

$$
R_{/ M}=\sum_{\substack{i<j \\ i, j=1}}^{n} K_{/ M}\left(e_{i}, e_{j}\right)
$$

где $e_{i}$ - ортонормированньй базис касательного пространства к $F$.

СледСТВИЕ. Пусть $f: N^{n} \rightarrow M^{n+1}, n \geqslant 2,-$ регулярное класса $C^{2}$ погружение компактного связного многообразия $N^{n}$ в полное односвязное риманово многообразие неположительной секиионной кривизны. Если внешняя кривизна Риччи поверхности $F=f\left(N^{n}\right)$ в каждой точке неотричательна, а внешняя скалярная кривизна положительна, то $F=f\left(N^{n}\right)$ - гиперповерхность, диффеоморфная cфере.

ДокАЗАТЕЛЬСтво. Из условия на внешнюю кривизну Риччи следует, что вторая квадратичная форма в каждой точке при соответствующем выборе нормали неположительно определена [12]. Из положительности внешней скалярной кривизны и из формулы Гаусса следует, что ранг второй квадратичной формы $\geqslant 2$. Применяя теорему, мы получим требуемое утверждение.

\section{СПИСОК ЦИТИРОВАННОЙ ЛИТЕРАТУРЫ}

[1] Стенберг С. Лекции по дифференциальной геометрии. М.: Мир, 1970.

[2] Chern S.S., Lashof R. K. The total curvature of immersed manifolds // Amer. J. Math. 1957. V. 79. P. 308-318.

[3] Heijenoort S. On locally convex manifolds // Communications on Pure and Applied Mathematics. 1952. V. 5. P. 223-242.

[4] Currier C. On hypersurfaces of Hyperbolic space infinitesimally supported by horospheres // Trans. Amer. Math. Soc. 1989. V. 313. №1. P. 420-431.

[5] Борисенко А. А., Власенко Д. И. Выпуклые поверхности в пространстве Лобачевского // Математ. физика, анализ, геометрия. 1997. Т. 4. № 3. С. 278-285.

[6] Бураго Ю. Д., Залгаллер В. А. Введение в риманову геометрию. М.: Наука, 1994.

[7] Бураго Ю. Д., Залгаллер В. А. Выпуклые множества в римановых пространствах // Успехи матем. наук. 1977. Т. 47. № 2. С. 3-5.

[8] Gromov M. Lectures on manifolds of nonpositive curvature. Boston, 1985.

[9] Alexander S. Locally convex hypersurfaces of negatively curved spaces // Proc. AMS. 1977. V. 64. № 2. P. 321-325.

[10] Борисенко А. А. Внешняя геометрия сильно параболических многомерных подмногообразий // Успехи матем. наук. 1997. Т. 52. №6(318). С. 3-52.

[11] Warner F. W. Extensions of the Rauch comparison theorem to submanifolds // Trans. Amer. Math. Soc. 1966. V. 122. № 2. P. 341-356.

[12] Грей А. Трубки. М.: Мир, 1993. 